\title{
Caracterización de la actividad demostrativa. Una experiencia en secundaria
}

Autores: BOLIVAR SÁNCHEZ, Cristina Antonia. MARTIN BAQUERO, Mayerly Andrea.

Palabras Claves: Actividad demostrativa, exploración, visualización, formulación de conjeturas, verificación, explicación, prueba, demostración, uso de definición en demostración.

\section{Descripción}

En este trabajo se recapitula el proceso y los resultados obtenidos de una experiencia de aprendizaje desarrollada con estudiantes de grado noveno del Instituto Pedagógico Nacional, en la que se realizó una caracterización de la actividad demostrativa asociada a un problema geométrico abierto, haciendo uso de un software de geometría dinámica. Se tomó como referencia la conceptualización propuesta por el grupo Aprendizaje y Enseñanza de la Geometría de la Universidad Pedagógica Nacional.

\section{Contenidos}

En el trabajo se presentan siete capítulos y una sección de anexos.

El capítulo 1 presenta la justificación del estudio, la problemática que se abordó y los objetivos planteados. El capítulo 2, da cuenta de la revisión de la literatura que se realizó. En el Capítulo 3, se precisan los conceptos en torno a la actividad demostrativa que hacen parte del marco teórico del estudio. En el Capítulo 4, se realiza una descripción del contexto de la población en el que se llevó a cabo la investigación; se presenta uno de los problemas propuestos a los estudiantes en el estudio, el proceso seguido para la selección del problema que posteriormente se analizó, y se realiza una descripción de las fases del análisis de la información. El Capítulo 5 presentan los indicadores y la caracterización realizada a partir de la implementación del problema. En el Capítulo 6 se muestra la síntesis realizada de los elementos que constituyen las acciones de la actividad demostrativa que se evidenciaron en los estudiantes, así como un contraste entre las características de la actividad demostrativa de estudiantes universitarios y estudiantes de básica secundaria y finalmente el Capítulo $7 \mathrm{da}$ cuenta de las conclusiones del estudio. Para complementar y sustentar la información que se presenta en el Capítulo 4 se incluye al final de este trabajo, después de las referencias, dos secciones de anexos, donde se presentan los problemas que se diseñaron e implementaron y el listado de definiciones y teoremas en el que se basaron los estudiantes para dar solución a los problemas.

\section{Metodología}

Para el desarrollo del presente trabajo se establecieron varios momentos:

En un primer momento se realizó la revisión de la literatura que consideramos presentaban aspectos similares a los que pretendíamos desarrollar, destacando la metodología y los resultados que permitirían contrastarlos con los resultados que obtuvieron en este estudio.

En un segundo momento se consolidó el marco teórico sobre el que basaríamos 
nuestro estudio, tomando como referencia el elaborado por el grupo Enseñanza y Aprendizaje de la Geometría de la Universidad Pedagógica Nacional.

En un tercer momento se realizó una revisión del plan de estudios y la caracterización de la aproximación metodológica de las clases de geometría de los estudiantes de grado noveno del Instituto Pedagógico Nacional.

En un cuarto momento se seleccionaron cuatro problemas que se propondrían a los estudiantes, realizando para ello la revisión de algunas actividades extraídas de otras investigaciones, y eligiendo aquellos problemas encaminados a que los estudiantes identificaran propiedades comunes al rectángulo y al trapecio isósceles. Estos problemas fueron mostrados al grupo de Aprendizaje y Enseñanza de la Geometría, buscando identificar las dificultades que podía tener cada problema en cuanto a la interpretación de éstos, la formulación de la conjetura y su posterior demostración, para finalmente reformularlos y presentarlos a los estudiantes.

En un quinto momento se implementaron los cuatro problemas en cinco sesiones, con un grupo de seis estudiantes, en horas extraclase. De este proceso se recogieron las grabaciones de audio y video y se realizó la transcripción de cada una de ellas. Se eligieron las transcripciones correspondientes a uno de los problemas, agrupándolas por fragmentos de acuerdo a la acción propia de la actividad demostrativa al que respondiera cada uno de ellos, para su posterior análisis. Del análisis realizado se presenta una síntesis de los aspectos más sobresalientes, así como de las dificultades evidenciadas y se hace una comparación entre la actividad demostrativa que desarrollan estudiantes universitarios y estudiantes de básica secundaria.
Finalmente, en un sexto momento se muestran las conclusiones a la luz de los resultados obtenidos, teniendo presente tres aspectos particulares: el primero corresponde al ambiente de aprendizaje, el segundo a los problemas y el tercero al uso de la geometría dinámica.

\section{Conclusiones}

Las conclusiones que se presentan en este trabajo están relacionadas con el interés que orientó este estudio, con respecto a la caracterización de la actividad demostrativa de estudiantes de básica secundaria a partir de la solución de un problema geométrico abierto. Se señalan en ellas los logros más destacados de los estudiantes en cada una de las acciones de la actividad demostrativa en las que se centró el estudio y se mencionan algunas dificultades en torno a las limitaciones del diseño del mismo. Con respecto al ambiente de aprendizaje, cabe destacar que se logró favorecer un ambiente que permitió a los estudiantes participar activamente en la construcción social del conocimiento, en la que se pasó de ser asumido exclusivamente por el profesor como responsable del proceso enseñanza y aprendizaje a ser una responsabilidad compartida, en la que los estudiantes tenían un papel activo en dicho proceso. Una experiencia de este tipo puede enriquecerse realizando algunas modificaciones como: revisar y explicar con detalle las definiciones y teoremas que harán parte del sistema, aunque se asuma que han sido trabajados previamente. Al escoger los problemas que se le proponen a los estudiantes hay que tener en cuenta que la demostración de las conjeturas que surgen no implique observar más de un elemento a la vez, pues esto puede desviar su atención en lo que deben demostrar, y llevarlos a que se concentren en hechos no necesariamente relevantes para la demostración. 
Con respecto a los problemas, se pudo determinar que los estudiantes tienen mayor facilidad para elegir argumentos aunque no necesariamente sean los correctos, que para construirlos. El nivel argumentativo de los estudiantes permite explicar las diferencias en sus respuestas, ya que los que presentan un nivel más bajo le encontraban poco sentido a una demostración, por lo que casi siempre elegían argumentos empíricos; en cambio, los que cuentan con un mayor nivel, reconocen la generalidad de la prueba y su papel en el establecimiento de la verdad de una afirmación y por tanto, son mejores en la construcción de una demostración y en la selección de argumentos.

Con respecto al uso de la geometría dinámica, el programa aportó a la acción de visualizar, lo que posibilitó que los estudiantes completaran la conjetura del segundo problema. No se puede concluir sobre los efectos de la geometría dinámica en la construcción de una justificación; lo anterior se explica tal vez por el hecho de que en el diseño metodológico no se previó que el manejo que tenían los estudiantes de Cabri no era suficiente para permitirles a los estudiantes aprovechar la opción de arrastre, para realizar exploraciones, verificar y visualizar hechos geométricos, que les permitieran vislumbrar un camino para la justificación.

A pesar del hecho antes mencionado, no debe desestimarse el papel de la geometría dinámica al crear un ambiente propicio para la indagación y de construcción colectiva de las justificaciones, ya que permitió que los estudiantes participaran en la solución de un problema abierto involucrándose de manera genuina en la solución y justificación del problema 